\title{
Synthesis of 4' $\alpha$-C Phenyl-Branched Carbocyclic Nucleoside Using Ring-Closing Metathesis
}

\author{
Joon Hee Hong ${ }^{\star}$ and Ok Hyun Ko \\ College of Phamacy, Chosun University, Gwangiu 507-759, Korea \\ Received May 28,2003
}

\begin{abstract}
An efficient synthetic route for preparing novel $4^{1} \alpha-C$ phenyl branched carbocyclic nucleoside is deseribed. The installation of phenyl group at the 4'-position of carbocyclic nucleoside was successfully accomplished via a scquential [3,3]-sigmatropic reartangement and ring-closing metathesis (RCM) beginning from simple ketone such as 2-hydroxy acelophenone.
\end{abstract}

Key Words : Carbocyclic nucleoside, Antiviral agents, [3,3]-Sigmatropic rearrangement, Ring-closing metathesis

\section{Introduction}

Carbocyclic nucleosides are a group of compounds that are structurally similar to natural nucleosides where the furanose oxygen is replaced by a methylene group. The replacement of the furanose ring oxygen by carbon is of particular interest because the resulting carbocyclic nucleosides possess a greater metabolic stability to phosphorylase. ${ }^{1}$ which cleaves the glycosidic bond of nucleosides. Since the cyclopentane ring of carbocyclic nucleosides can emulate the furanose moiety, a number of these compounds exhibit interesting biological activities, particularly in the areas of antivital and anticancer chemotherapy. The recent discovery of abacavir ${ }^{2}$ as an anti-HIV agent has given a strong impetus to the search of novel nucleosides in this class of compounds.

Recently, several branched-nucleoside ${ }^{3}$ have been synthesized and evaluated as potent antitumor or antiviral agents. Among them, $4^{\prime} \alpha$-C'-ethenyl, ${ }^{4} 4^{\prime} \alpha$ - $C^{\prime}$-ethynyl ${ }^{5}$ and $4^{\prime} \alpha-C^{\prime}-$ cyano ${ }^{6}$ nucleosides, which have an additional double or triple bond at the 4'-position, were reported to exhibit potent antivital and antitumor activities.

Encouraged by these interesting structures and antiviral activities, novel class of nucleoside comprising branched carbocyclic nucleosides with an additional phenyl group at 4'-position was synthesized (Figure 1).

It is well known that the [3.3]-sigmatropic rearrangement.

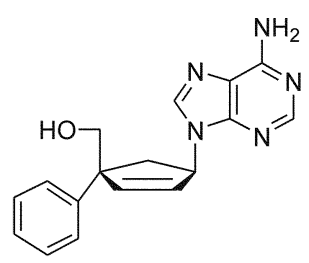

12

Figure 1

"Corresponding author: Tel.: +82-62-230-6378; Гax: +82-62222-5414: t-mail: hongih@mail.chosun.ac.kr
$\mathrm{RCM}^{8}$ and $\mathrm{Pd}(0)$ catalyzed allylic alkylation have been employed widely in synthetic organic chemistry. A very convenient and general synthetic procedure for nucleosides using these procedures is described in this paper.

\section{Results and Discussion}

Although the synthetic methods of $4^{\prime} \alpha-C$ alkyl branched furanose ${ }^{10}$ and carbocyclic nucleosides ${ }^{11}$ have been reported. the branches were limited to only alkyl groups $\left(\mathrm{C}_{3}, \mathrm{C}_{9} \mathrm{H}_{19}\right.$, Benzyl) with use of nucleophilic alkyl substitution reactions. Thus far, no synthetic example of 4'-phenyl branched nucleosides has been reported in the literature. The dearth of examples may be due to the synthetic difficulties for elaborating a necessary quaternary carbon containing phenyl group. As shown in Scheme 1, it was envisioned that the ring-closing metathesis of proper diolefin 7 , which could be readily synthesized via a sequential $[3,3]$-sigmatropic rearrangement and carbonyl addition starting from a simple acyclic precursor, the 2 -hydroxy ketone derivative 1 , would produce phenyl branched cyclopentene $\mathbf{8}$ as the key intermediates.

The protection of the hydroxyl of commercially available starting material 1 with TBDMSCl followed by the HornerWadsworth-Emmons (HWE) reaction ${ }^{12}$ provided the $\alpha \beta$ unsaturated ethyl ester $\mathbf{3}$ as cisinons isomeric mixtures. It was unnecessary to separate these isomers, because they were merged into one isomer in subsequent reaction. Ester 3 was reduced to the allylic alcohols $\mathbf{4}$ using diisobutylaluminum hydride (DIBALH) in high yield, which were subjected to a normal Johnson's orthoester Claisen rearrangement ${ }^{7}$ using triethyl orthoacetate to give $\gamma \delta$-unsaturated ester 5 in $81 \%$ yield. The slow addition of DIBALH to a solution of the esters 5 in toluene at $-78^{\circ} \mathrm{C}$ furnished the desired aldehyde 6 , which was subjected to carbonyl addition by $\mathrm{CH}_{2}-\mathrm{CHMgBr}$ to yield the olefin 7 as inseparable diastereomeric mixtures.

Without separation, each diolefin 7 , was subjected to the standard ring-closing metathesis ${ }^{8}$ conditions using [benzy]idene-bis(tricyclohexylphosphine)-dichlororuthenium] to 

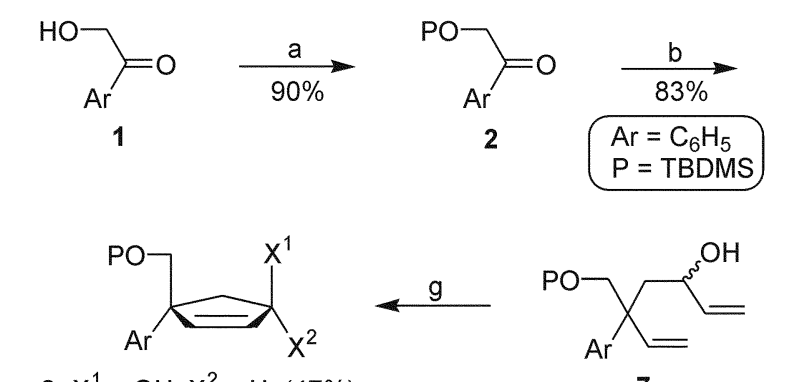<smiles>CCOC(=O)C=C([Al])CO</smiles>
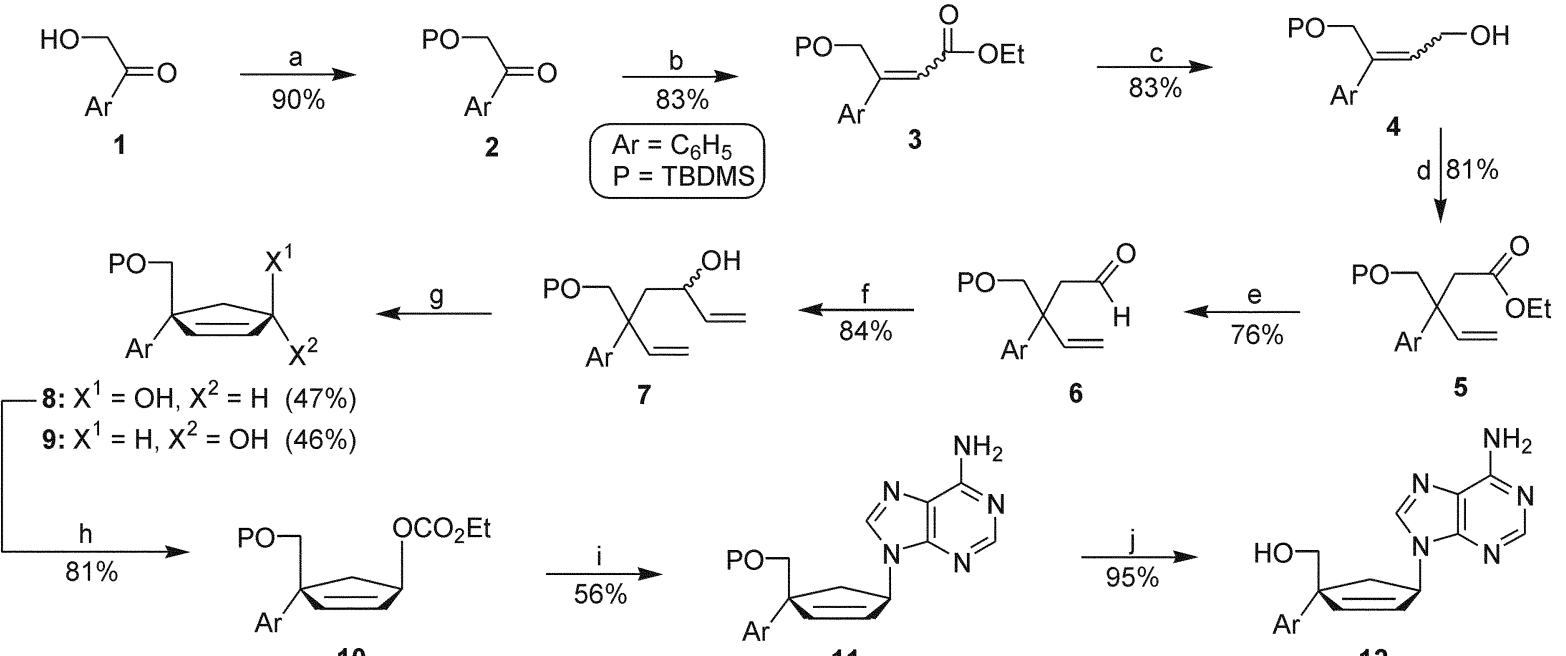

11

12

Scheme 1. Reagents: a) TBDMSCI, $\mathrm{CH}_{2} \mathrm{Cl}_{2}$. imidazole. 0 "C. $5 \mathrm{ht}$ b) Triethylphosphonoacelate. NaH. THF. rt. 1 h; c) IDIBAI.H, CH

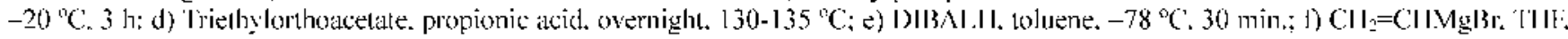

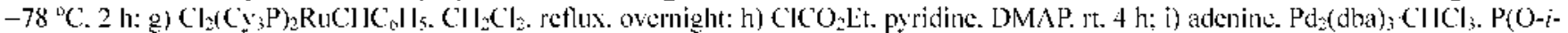
Pr). NaH. THF/DMSO. retlux. overnight: j) TBAF. THF. rt. $5 \mathrm{~h}$.

provide the cyclopentenols 8 and 9 , respectively. The stereochemical assignments were made on the basis of NOE experiments. On irradiation of $\mathrm{C}_{\mathrm{J}}-\mathrm{H}$. NOE was observed at the methylene protons of the hydroxymethyl group of 9 . but not at the methylene protons those of 8 .

In order to couple the cyclopentenol with the adenine base using a routine nucleophilic substitution type reaction, the $\alpha$ isomers 9 was subjected to a mesylation reaction $(\mathrm{MsCl}$. TEA, $\left.\mathrm{CH}_{2} \mathrm{Cl}_{2}\right)^{1 .}{ }^{1.3}$ Unexpectedly, the reaction had a very low yield $(10-20 \%)$ and was irreproducible. Therefore, our attention was turned to a Trost protocol. ${ }^{9}$ Palladium( $(0)$-catalyzed reactions have played the central role in allylic functionalization. This methodology has been successfully applied to synthesizing the desired nucleosides. The cyclopentenol 8 was activated to $\mathbf{1 0}$ using ethyl chloroformate in a high yield $(81 \%)$, which was coupled with an adenine anion generated by $\mathrm{NaH} / \mathrm{DMSO}$ using the well-known coupling catalyst [tris(dibenzylidene-acetone)-dipalladium( $(0)$-chloroform] adduct to give 11. The required stereochemistry of nucleoside 11 was successfully controlled from the $\beta$-configuration of 10 via $\mathrm{Pd}(0)$ catalyzed $\pi$-allyl complex mechanism." Although a sinall amount of the $N 7$-regioisomer ${ }^{14}(<7 \%)$ was observed, the compound 11 and its $N 7$-isomer could be easily separated. The desilylation of 11 was performed by a treatment with tetrabutylammonium fluoride (TBAF) to give the final nucleoside $\mathbf{1 2}$ in a $95 \%$ yield.

Based on extensive literature searching, the compound 12 appears to be a novel nucleoside. Antiviral evaluations against various viruses such as HIV-I, HSV-I, HSV-2 and HCMV were performed. However, it did not show any significant activity or cytotoxicity up to $100 \mu \mathrm{M}$.

In summary, a short and concise synthetic method for synthesizing 4' $\alpha-C$ phenyl branched carbocyclic nucleosides from a simple $\alpha$-hydroxy ketone derivative was developed. Our procedure highlights the simplicity and efficiency in the installation of phenyl branch at cyclopentene ring systems.
On the basis of this strategy, the enantiomeric syntheses of branched nucleosides with different nucleobases and substituents are in progress.

\section{Experimental Section}

All chemicals were reagent grade and were used as purchased. All moisture-sensitive reactions were performed in an inert atmosphere of either $\mathrm{N}_{2}$ or Ar using distilled dry solvents. The elemental analyses were performed by Elemental Analyzer System (Profile HV-3). NMR spectra were recorded on a bruker 300 Fourier transform spectrometer.

2-(t-Butyldimethylsilyloxy)-acetophenone (2): TBDMSCl $\left(22.4 \mathrm{~g}, 0.15 \mathrm{~mol}\right.$ ) was added slowly at $0^{\circ} \mathrm{C}$ to a solution of 2-hydroxy acetophenone 1 ( $18.5 \mathrm{~g}, 0.135 \mathrm{~mol}$ ) and imidazole $(13.5 \mathrm{~g}, 0.203 \mathrm{~mol})$ in $\mathrm{CH}_{2} \mathrm{Cl}_{2}(300 \mathrm{~mL})$, and stirred for $5 \mathrm{~h}$ at the same temperature. The solvent was evaporated under a reduced pressure. The residue was extracted twice with diethyl ether and water. The combined organic layer was dried over anhydrous $\mathrm{MgSO}_{4}$, filtered, and concentrated under a reduced pressure. The residue was purified by silica gel column chromatography (EtOAc/hexane, I: 10 ) to give compound 2 (32.5 g. $90 \%$ ) as a colorless oil: 'H NMR $\left(\mathrm{CDCl}_{3}, 300 \mathrm{MHz}\right) \delta 7.80(\mathrm{~d}, J-7.2 \mathrm{~Hz}, 2 \mathrm{H}), 7.46-7.30(\mathrm{~m}$, $3 \mathrm{H}), 4.79(\mathrm{~s}, 2 \mathrm{H}), 0.80(\mathrm{~s}, 9 \mathrm{H}),-0.01(\mathrm{~s}, 6 \mathrm{H}) ;{ }^{13} \mathrm{C} . \mathrm{NMR}$ (CDCli) $\delta 197.42,134.85,133.22,128.56,127.83,67.40$, $25.78,18.44,-5.36 ;$ Anal calc for $\mathrm{C}_{1,4} \mathrm{H}_{22} \mathrm{O}_{2} \mathrm{Si}: \mathrm{C}, 67.15 ; \mathrm{H}$, 8.86. Found: C, 66.97; H. 8.65 .

(E) and (Z)-4-( $t$-Butyldimethylsilyloxy)-3-phenyl-but2-enoic acid ethyl ester (3): To a suspension of sodium hydride (60\% in mineral oil, $0.74 \mathrm{~g}, 18.5 \mathrm{mmol}$ ) in distilled THF at $0{ }^{\circ} \mathrm{C}$, triethyl phosphonoacetate $(2.81 \mathrm{~mL}, 18.5$ mmol) was added drop wise and with constant stirring at room temperature for $1 \mathrm{~h}$. The ketone 2 (4.6 g. $18.5 \mathrm{mmol}$ ) was added to this mixture and stirred for $\mathrm{I}$ h. The solution 
was neutralized with $\mathrm{AcOH}$, and extracted with EtOAc. The organic layer was washed with brine and dried over anhydrous $\mathrm{MgSO}_{4}$, filtered and evaporated. The residue was purified by silica gel column chromatography (EtOAc/ hexane $1: 15)$ to give $3(4.9 \mathrm{~g} .83 \%)$ as a colorless oil: ${ }^{1} \mathrm{H}$ NMR $\left(\mathrm{CDCl}_{\hat{j}+} 300 \mathrm{MHz}\right)$ as mixture $\delta 7.39-7.04(\mathrm{~m} .5 \mathrm{H})$. $6.09,5.93(\mathrm{dt} J=1.3,1.8 \mathrm{~Hz}, 1 \mathrm{H}), 5.08,4.23$ (dd. $J=0.9$. 2. $1 \mathrm{~Hz} .2 \mathrm{H}$ ) 3.90 (dq. $J=6.9 .6 .9 \mathrm{~Hz} .2 \mathrm{H}) .1 .20 .0 .95$ (dt. $J$ $=6.9 .6 .9 \mathrm{~Hz} .3 \mathrm{H}), 0.84 .0 .65$ (s. s. $9 \mathrm{H}), 0.02 .-0.10$ (s. s. $6 \mathrm{H}$ ): Anal calc for $\mathrm{C}_{18} \mathrm{H}_{38} \mathrm{O}_{3} \mathrm{Si}: \mathrm{C} .67 .46: \mathrm{H}, 8.81$. Found: $\mathrm{C}$. 67.66: H. 8.95.

(E) and (Z)-t-(t-Butyldimethylsilyloxy)-3-methyl-but2-en-1-ol (4): To a solution of $3(8.9 \mathrm{~g} .27 .9 \mathrm{mmol})$ in $\mathrm{CH}_{2} \mathrm{Cl}_{2}(300 \mathrm{~mL})$. DIBALH $(58.55 \mathrm{~mL}, 1.0 \mathrm{M}$ solution in hexane) was added slowly at $-20^{\circ} \mathrm{C}$. and stirred for $\mathrm{l} h$ at the same temperature. To the resulting mixture. methanol (50 $\mathrm{mL}$ ) was added. The mixture was stirred at room temperature for $3 \mathrm{~h}$, and the resulting solid was filtered through a Celite pad. The filtrate was concentrated under vacuum and the residue was purified by silica gel column chromatography (EtOAc/hexane. $1: 5$ ) to give alcohol + (6.4 g. $83 \%$ ) as a colorless oil: ' $\mathrm{H} \mathrm{NMR}\left(\mathrm{CDCl}_{3}, 300 \mathrm{MHz}\right)$ as mixture $\delta 7.32-7.07(\mathrm{~m} .5 \mathrm{H}) .5 .99 .5 .91$ (dt. $J=6.6 .6 .6 \mathrm{~Hz}$. $1 \mathrm{H}) .4 .31$ (d. $J=6.6 \mathrm{~Hz}, 2 \mathrm{H}), 4.27$ (s. $2 \mathrm{H}), 0.85,0.81$ (s. s. $9 \mathrm{H}), 0.02(\mathrm{~m}, 6 \mathrm{H}):$ Anal calc for $\mathrm{C}_{16} \mathrm{H}_{26} \mathrm{O}_{3} \mathrm{Si}: \mathrm{C} .69 .01: \mathrm{H}$. 9.41. Found: C. 69.18: H. 9.26 .

( \pm )-3-(t-Butyldimethylsilyloxymethyl)-3-phenyl-pent-4enoic acid ethyl ester (5): A solution of allylic alcohol 4 (19.3 g. $69.32 \mathrm{mmol}$ ) in triethyl orthoacetate $(300 \mathrm{~mL}$ ) and $0.9 \mathrm{~mL}$ of propionic acid was heated at $130-135^{\circ} \mathrm{C}$ overnight with stirring to allow for the removal of ethanol. The excess of triethyl orthoacetate was removed by distillation and the residue was purified by silica gel column chromatography (EtOAc/hexane. $1: 15)$ to give $5(19.6 \mathrm{~g} .81 \%$ ) as a colorless oil: ${ }^{l} \mathrm{H} N M R\left(\mathrm{CDCl}_{\hat{3}} .300 \mathrm{MHz}\right) \delta 7.36-7.25(\mathrm{~m}, 5 \mathrm{H}), 6.26$ (dd. $J=18.0 .11 .1 \mathrm{~Hz} .1 \mathrm{H}$ ). 5.31 (dd. $J=11.4 .1 .2 \mathrm{~Hz}$. IH). $5.16(\mathrm{dd}, J=17.7 .0 .6 \mathrm{~Hz}, \mathrm{IH}), 4.10-3.99(\mathrm{~m} .+\mathrm{H}) .3 .00(\mathrm{~s}$. $2 \mathrm{H}$ ). $1.18(\mathrm{t} . J=6.9 \mathrm{~Hz} .3 \mathrm{H}$ ). 0.99 (s. $9 \mathrm{H}), 0.02$ (two s. $6 \mathrm{H}$ ): ${ }^{13} \mathrm{C} \mathrm{NMR}\left(\mathrm{CDCl}_{3}\right) \delta 171.51,143.17,1+2.33 .127 .82,127.34$ 126.30, 114.34. 67.73, 59.94, 48.70, 39.74, 25.76, 18.19 . 14.07. -5.71: Anal calc for $\mathrm{C}_{20} \mathrm{H}_{32} \mathrm{O}_{3} \mathrm{Si}$ : C. 68.92: $\mathrm{H}, 9.25$. Found: C. $68.69: \mathrm{H}, 9.05$

( \pm )-3-( $t$-Butyldimethylsilyloxymethyl)-3-phenyl-pent-4enal (6): To a solution of $5(6.7 \mathrm{~g} .19 .2 \mathrm{mmol})$ in toluene (200 mL). DIBALH (14.1 mL, 1.5 M solution in toluene) was added slowly at $-78{ }^{\circ} \mathrm{C}$. and stirred for $30 \mathrm{~min}$. at the same temperature. To the mixture. methanol ( $50 \mathrm{~mL}$ ) was added. The mixture was stirred at room temperature for $2 \mathrm{~h}$. and the resulting solid was filtered through a Celite pad. The filtrate was concentrated under vacuum and the residue was purified by silica gel column chromatography (EtOAc/ hexane $1: 15)$ to give $6(4.4 \mathrm{~g}, 76 \%)$ as a colorless oil: ${ }^{1} \mathrm{H}$ NMR $\left(\mathrm{CDCl}_{\hat{\jmath} .} 300 \mathrm{MHz}\right) \delta 9.63$ (s. IH). $7.3+-7.26(\mathrm{~m} .5 \mathrm{H})$. 6.09 (dd. $J=17.7 .11 .1 \mathrm{~Hz}, 1 \mathrm{H}) .5 .34$ (d. $J=11.1 \mathrm{~Hz}, \mathrm{IH})$. 5.16 (d. $J=17.4 \mathrm{~Hz}, \mathrm{IH}$ ). 3.86 (s. $2 \mathrm{H}$ ). 2.97 (dq. $J=16.2$. 3.0). 0.88 (s. 9H) -0.01 (s. $6 \mathrm{H}):{ }^{13} \mathrm{C} \mathrm{NMR}\left(\mathrm{CDCl}_{3}\right) \delta$ 202.86, 142.38, 141.49, 128.38, 127.33, 126.83, 115.70 .
69.28, 49.01, 25.76, 18.19, -5.74: Anal calc for $\mathrm{C}_{18} \mathrm{H}_{28} \mathrm{O}_{2} \mathrm{Si}$ C. 71.00: H. 9.27. Found: C. 71.34: H, 9.15.

$( \pm)-(3 R$ and $3 S, 5 S)-5$-( $($-Butyldimethylsilyloxymethyl)5-phenyl-hepta-1,6-dien-3-ol (7): To a cooled $\left(-78{ }^{\circ} \mathrm{C}\right)$ solution of $6(7.0 \mathrm{~g} .23 .1 \mathrm{mmol})$ in dy THF $(120 \mathrm{~mL})$ vinylmagnesium bromide $(27.7 \mathrm{~mL}, 1.0 \mathrm{M}$ solution in THF) was added slowly. After $2 \mathrm{~h}$, a saturated $\mathrm{NH}_{4} \mathrm{Cl}$ solution (23 $\mathrm{mL}$ ) was added and the reaction mixture was warmed slowly to room temperature. The mixture was extracted with EtOAc $(2 \times 150 \mathrm{~mL})$. The combined organic layer was dried over $\mathrm{MgSO}_{4}$. filtered and evaporated. The residue was purified by silica gel column chromatography (EtOAc/ hexane. $\mathrm{L}: 10)$ to give $7(6.4 \mathrm{~g} .84 \%)$ as a colorless oil: ${ }^{1} \mathrm{H}$ $\mathrm{NMR}\left(\mathrm{CDCl}_{2}, 300 \mathrm{MHz}\right) \delta 7.36-7.21(\mathrm{~m} .5 \mathrm{H}), 6.02-5.96(\mathrm{~m}$. $2 \mathrm{H}) .5 .21-4.96(\mathrm{~m}, 4 \mathrm{H}), 4.1 \mathrm{l}-3.89(\mathrm{~m}, 2 \mathrm{H}) .2 .2 \mathrm{l}-2.07(\mathrm{~m}$. 2H). $0.88(\mathrm{~m}, 9 \mathrm{H}), 0.04(\mathrm{~m}, 6 \mathrm{H})$ : Anal calc for $\mathrm{C}_{2}\left(\mathrm{H}_{32} \mathrm{O}_{2} \mathrm{Si}\right.$ : C. $72.23:$ H. 9.70. Found: C. $72.31:$ H. 9.88 .

( \pm )-(1R,4S)-4-(t-Butyldimethylsilyloxymethyl)-t-phenylcyclopent-2-enol $(8)$; and $( \pm)-(1 S, 4 S)-4$ - $(t$-Butyldimethylsilyloxymethyl)-4-phenyl-cyclopent-2-enol (9): To a solution of $7(3.1 \mathrm{~g} .9 .24 \mathrm{mmol})$ in $\mathrm{dny} \mathrm{CH}_{2} \mathrm{Cl}_{2}(20 \mathrm{~mL})$ Grubbs catalyst $(0.76 \mathrm{~g} 0.92 \mathrm{mmol})$ in dry $\mathrm{CH}_{2} \mathrm{Cl}_{2}(10 \mathrm{~mL})$ was added slowly over a 10 -minute period under a $\mathrm{N}_{2}$ atmosphere. The reaction mixture was refluxed overnight. and cooled to room temperature. The mixture was concentrated under vacuum, and the residue was purified by silica gel column chromatography (EtOAc/hexane. $1: 5$ ) to give the cyclopentenols. $8(1.32 \mathrm{~g} .47 \%)$ and $9(1.3 \mathrm{~g} .46 \%)$. as colorless oils. respectively: Compound 8: ${ }^{1} \mathrm{H}$ NMR $\left(\mathrm{CDCl}_{3}\right.$. $300 \mathrm{MHz}) \delta 7.28-7.12(\mathrm{~m} .5 \mathrm{H}) .6 .03-5.97(\mathrm{~m}, 2 \mathrm{H}), 4.60-$ $4.53(\mathrm{~m}, \mathrm{lH}), 3.65(\mathrm{~d}, J=9.6 \mathrm{~Hz}, \mathrm{lH}), 3.50(\mathrm{~d}, J=9.6 \mathrm{~Hz}$. IH). 2.33 (dd, $J=13.8 .6 .9 \mathrm{~Hz}, \mathrm{lH}$ ), 2.12 (dd. $J=8.1,6.9$ Hz. $\mathrm{IH}), 0.81$ (s. 9H) $-0.0 \mathrm{l}$ (s. 6H): ${ }^{13} \mathrm{C} \mathrm{NMR}\left(\mathrm{CDCl}_{3}\right) \delta$ 145.04, 136.44, 135.53. 128.46, 126.61.75.62,69.77, 58.70. 45.73, 26.00, 18.62, -5.41: Anal calc for $\mathrm{C}_{18} \mathrm{H}_{28} \mathrm{O}_{2} \mathrm{Si}: \mathrm{C}$. 71.00: H, 9.27. Found: C. 70.73: H. 9.08. Compound 9: ${ }^{1} \mathrm{H}$ NMR $\left(\mathrm{CDCl}_{2}, 300 \mathrm{MHz}\right) \delta 7.24-7.19(\mathrm{~m}, 5 \mathrm{H}) .6 .12(\mathrm{~d}, J=$ $4.8 \mathrm{~Hz}, \mathrm{lH}), 5.93(\mathrm{dd} . J=6.0 .2 .1 \mathrm{~Hz}, \mathrm{lH}), 4.87(\mathrm{~s}, \mathrm{lH}), 3.55$ (s. $2 \mathrm{H}$ ), 2.70 (dd, $J=13.2,7.2 \mathrm{~Hz}, \mathrm{IH}$ ). 1.83 (dd. $J=18.0$. $4.8 \mathrm{~Hz}, \mathrm{lH}), 0.75$ (s. 9H) $-0.13,-0.15$ (s. s. $6 \mathrm{H}):{ }^{13} \mathrm{C}$ NMR $\left(\mathrm{CDCl}_{3}\right) \delta 144.98,135.74 .135 .03,127.86,125.51$. 75.02. 68.12, 57.65, 43.23, 25.78, 18.14, -5.43 : Anal calc for $\mathrm{C}_{18} \mathrm{H}_{28} \mathrm{O}_{2} \mathrm{Si}: \mathrm{C} .71 .00: \mathrm{H}, 9.27$. Found: C. 71. 19: H. 9.11.

$( \pm)-(1 R,+S)-1-E$ thoxy carbonyloxy-t-(t-butyldimethylsilyloxymethyl)-t-phenyl-cyclopent-2-ene (10): To a solution of $8(4.4 \mathrm{~g} .14 .43 \mathrm{mmol})$ in anlydrous pyridine (50 $\mathrm{mL}$ ) ethyl chloroformate $(2.76 \mathrm{~mL} .28 .87 \mathrm{mmol})$ and DMAP $(0.17 \mathrm{~g} .1 .+\mathrm{mmol})$ was added. The reaction mixture was stirred for $4 \mathrm{~h}$ at room temperature. The reaction mixture was quenched with a saturated $\mathrm{NaHCO}_{3}$ solution $(2 \mathrm{~mL})$ and concentrated under vacuum. The residue was extracted with EtOAc. dried over $\mathrm{MgSO}_{4}$. filtered. and concentrated. The residue was purified by silica gel column chromatography (EtOAc/hexane. $1: 10$ ) to give $10(4.4 \mathrm{~g} .81 \%$ ) as a colorless șynu: ${ }^{l} \mathrm{H}$ NMR $\left(\mathrm{CDCl}_{3 .} 300 \mathrm{MHz}\right) \delta 7.41-7.31(\mathrm{~m}, 5 \mathrm{H})$. $6.56(\mathrm{~d}, J=5.7 \mathrm{~Hz} . \mathrm{IH}), 6.15(\mathrm{dd}, J=6.0 .2 .4 \mathrm{~Hz}, \mathrm{IH}) .5 .72$ (br d. $1 \mathrm{H}), 4.36(\mathrm{q} . J=7.5 \mathrm{~Hz}, 2 \mathrm{H}) .3 .93(\mathrm{~d}, J=9.3 \mathrm{~Hz}, \mathrm{lH}$ ). 
3.87 (d. $J=9.3 \mathrm{~Hz}, \mathrm{IH}) .2 .6+(\mathrm{dd}, J=1+.1 .7 .2 \mathrm{~Hz}, \mathrm{lH}) .2 .39$ (dd. $J=14.1 .3 .3 \mathrm{~Hz}, \mathrm{IH}$ ). 1.44 (t. $J=7.2 \mathrm{~Hz}, 3 \mathrm{H}$ ). 0.97 (s. $9 \mathrm{H}), 0.04$ (s. $6 \mathrm{H}):{ }^{13} \mathrm{C}$ NMR $\left(\mathrm{CDCl}_{3}\right) \delta 154.99,145.24$. 142.32 . 129.57, 128.06, 126.89. 126.22,82.88, 71.24. 63.78. 58.61, 40.97, 25.77, 18.20, 14.31, -5.61: Anal calc for $\mathrm{C}_{21} \mathrm{H}_{32} \mathrm{O}_{4} \mathrm{Si}$ : C. 66.98: H. 8.57. Found: C. 66.70: H. 8.60

( ()$\left.\left.\left.-\left(1^{\prime} R, 4^{\prime} S\right)-9-[4-(t-B u t y] d i m e t h y] s i l y\right] l o x y m e t h y\right]\right)-4-$ phenyl-cyclopent-2-en-1-yl] adenine (11): To a pure $\mathrm{NaH}$ (23.4 mg. $0.98 \mathrm{mmol}$ ) in anhỵdrous DMSO (3.4 mL) adenine $(13+\mathrm{mg} .0 .98 \mathrm{mmol}$ ) was added. The reaction mixture was stirred for $30 \mathrm{~min}$ at $50-55^{\circ} \mathrm{C}$ and cooled to room temperature. Simultaneously. $\mathrm{P}(\mathrm{O}-\mathrm{i}-\mathrm{Pr})_{3}(0.096 \mathrm{~mL}$. $0.22 \mathrm{mmol})$ was added to a solution of $\mathrm{Pd}_{2}(\mathrm{dba})_{3} \cdot \mathrm{CHCl}_{3}(4.6$ mg. $2.5 \mathrm{mmol})$ in anhydrous THF $(3.0 \mathrm{~mL})$. which was stirred for $40 \mathrm{~min}$. To the adenine solution of DMSO. a catalyst solution of THF and $10(331 \mathrm{mg} .0 .88 \mathrm{mmol}$ ) dissolved in anlyydrous THF ( $3 \mathrm{~mL}$ ) was added slowly. The reaction mixture was stirred overnight at a refluxing temperature and quenched with water $(2 \mathrm{~mL})$. The reaction solvent was removed under reduced pressure. The residue was purified by silica gel column chromatography $(\mathrm{MeOH} /$ $\left.\mathrm{CH}_{2} \mathrm{Cl}_{2} .1: 15\right)$ to give $11(207 \mathrm{mg} .56 \%)$ as a white solid: mp 189-192 ${ }^{\circ} \mathrm{C}$ : UV (MeOH) $\lambda_{\text {trax }} 261 \mathrm{~nm}^{\mathrm{l}} \mathrm{H} \mathrm{NMR}\left(\mathrm{CDCl}_{3}\right.$. $300 \mathrm{MHz}) \delta 8.3+(\mathrm{s} .1 \mathrm{H}), 8.00(\mathrm{~s} .1 \mathrm{H}), 7.36-7.22(\mathrm{~m}, 5 \mathrm{H})$. 6.43 (dd. $J=5.4 .2 .1 \mathrm{~Hz} . \mathrm{lH}$ ) 6.02 (br s. $3 \mathrm{H}$ ) 5.79 (t. $J=6.6$ $\mathrm{Hz}, \mathrm{IH}) .3 .84$ (d. $J=9.3 \mathrm{~Hz}, 1 \mathrm{H}), 3.79(\mathrm{~d}, J=9.3 \mathrm{~Hz}, \mathrm{IH})$. 2.91 (dd. $J=13.5 .8 .4 \mathrm{~Hz}, \mathrm{IH}) .2 .43$ (dd. $J=13.5 .6 .6 \mathrm{~Hz}$. $1 \mathrm{H}) .0 .86(\mathrm{~s} .9 \mathrm{H}), 0.01(\mathrm{~s} .6 \mathrm{H}):{ }^{13} \mathrm{C} \mathrm{NMR}\left(\mathrm{CDCl}_{\mathfrak{j}}\right) \delta 155.57$. 152.84, 149.91,144.49, 141.75, 138.87, 129.75, 128.46. 127.16, 119.71, 69.96, 59.29, 42.70, 25.94, 18.45, -5.37: Anal calc for $\mathrm{C}_{23} \mathrm{H}_{31} \mathrm{~N} \leqslant \mathrm{OSi}: \mathrm{C}, 65.52: \mathrm{H}, 7.41: \mathrm{N}, 16.6 \mathrm{l}$. Found: C. 65.77: H. 7.54: N. 16.65 .

( \pm )-(1'R,4'R)-9-[4-(Hydroxymethy])-4-pheny]-cyclopent2-en-1-yl] adenine (12): To a solution of 11 (291 mg. 0.69 mmol) in THF (10 mL). TBAF (1.04 mL. $1.0 \mathrm{M}$ solution in THF) at $0{ }^{\circ} \mathrm{C}$ was added. The mixture was stirred at room temperature for $5 \mathrm{~h}$, and concentrated. The residue was purified by silica gel column chromatography $(\mathrm{MeOH} /$ $\left.\mathrm{CH}_{2} \mathrm{Cl}_{3} . \mathrm{L}: 5\right)$ to give $12(201 \mathrm{mg} .95 \%)$ as a white solid: $\mathrm{mp}$ $218-220^{\circ} \mathrm{C}:$ UV $\left(\mathrm{H}_{2} \mathrm{O}\right) \lambda_{\text {max }} 261 \mathrm{~nm} .{ }^{1} \mathrm{H}$ NMR (DMSO-do. $300 \mathrm{MHz}$ ) $\delta 8.12$ (s. $\mathrm{lH}$ ) 8.11 (s. $1 \mathrm{H}) .7 .33-7.29$ (m. $5 \mathrm{H}$ ). 7.21 (br s. $2 \mathrm{H}), 6.44(\mathrm{dd} . J=5.4 .2 .1 \mathrm{~Hz}, \mathrm{lH}) .6 .08$ (dd. $J=$ $6.0 .2 .+\mathrm{Hz}, \mathrm{IH}), 5.60(\mathrm{dd}, J=8.1,6.0 \mathrm{~Hz}, 1 \mathrm{H}), 5.0 \mathrm{l}(\mathrm{t} . J=$ $5 .+\mathrm{Hz} . \mathrm{IH}) .3 .68-3.56(\mathrm{~m} .2 \mathrm{H}) .2 .70(\mathrm{dd} . J=13.8 .8 .4 \mathrm{~Hz}$. lH) 2.35 (dd. $J=13.5 .6 .0 \mathrm{~Hz}, \mathrm{IH}$ ): ${ }^{13} \mathrm{C}$ NMR (DMSO-do) $\delta$ 155.95, 152.25, 149.22, 145.40, 141.06, 138.85, 129.68 . 128.19. 126.56, 126.15, 68.47, 59.14. 41.98: Anal calc for $\mathrm{C}_{17} \mathrm{H}_{17} \mathrm{~N}_{3} \mathrm{O}: \mathrm{C}$. 66.43: H. 5.58: N. 22.79. Found: C. 66.70: H. 5.72: N. 22.98 .

Acknowledgements. We thank Dr. C.-K Lee (Korea Research Institute of Chemical Technology) for antiviral assays.

\section{References}

1. Stoeckler. J. D; Cambor C.: Parks. R. E.. Ir. Biochemistr 1980.
19. 102

2. (a) Daluge. S. M.: Good. S. S.: Faletto. M. B.: Miller. W. H.: St Clair. M. H.: Boone. L. R.: Tisdale. M.: Parry. N. R.: Reardon. T. E.: Dornsife. R. E.: Averett. D. R.: Krenitsky. T. A. Antinticrob. Agents Chentother. 1997. H1. 1082; (b) Dobkin, J. F. Infect. Med. 1999. 17,625

3. (a) Maag. H.: Rydzewski, R. M.: McRoberts, M. J.: Verheyden. J .P. H.: Prisbe. E. T. J. Med Chem 1992. 35. 1440: (b) Chen. M. S.: Suttmantr. R. T.: Papp. E.: Cannon. P. D.: McRobert. M. T.: Bach. C.: Copeland. W. C.: Wang. T. S. F. Biochentistry 1993. 32.6002 : (c) Maag. H.: Nelson. J. T: Rios-Steiner. J. L.: Priste, E. J. J. Med Chem. 1994, 37. 431: (d) Maguire, A. R: Meng. W. G.: Roberts. S. M. Willetts, A. J. J. Chom. Soc. Perkin Trans. 11993. 1795: (e) Jenkins, I. D.: Verheyden, J. P. H; Moffatt, J. G. J. Am. Chem. Soc. 1976. 98. 3346: (f) Guillerm. D.: Muzard. M.: Allart. B.: Guillerm. G. Bioorg. Hed. Chen. Lett. 1995. 5. 1455:(g) Crich. D.: Hao. X. J. Org. Chent 1999. 64. 4016: (h) Crich. D. Hao. X. J. Org Chem. 1998, 63, 3796; (i) Jung. M. E.: Toyota. A. J. Org. Chem. 2001, 66. 2624: (j) Brown, B.; Hegedus, L .S. J. Org. Chem. 2000. 65, 1865: (k) Hegedus. L. S.: Geisler, L.: Riches. A. G.: Salmant. S. S.: Unmbricht. G. J. Org. Chem 2002. 67. 7649: (I) Hegedus. L. S.: Hervert. K. L.: Matsui. S. J. Ong. Chent. 2002. 67. 4076 .

4. Sugimoto. I.; Shuto, S.; Mori, S.: Shigeta. S.; Matuda, A. Bioong. 1fed Chem. Lett 1999.9.385.

5. (a) Nomura, M: Shuto. S: Tanaka, M.: Sasaki, T: Mori. S: Shigeta. S.: Matsuda. A. J. 1 fed Chem 1999. 42. 2901 (b) Ohrui. H.: Kohgo. S.: Kitano. K.: Sakata. S.: Kodama. E.: Yoshimura. K.: Matsuoka. M.: Shigeta. S.: Mitsuya. H. J. Med Chen. 2000. 13. 4516.

6. OYang. C.: Wu. H. Y; Fraser-Smith, E. B; Walker. K. A. M. Tetrahedron Lett. 1992, 33. 37

7. For selected references for nucleosides: (a) Ko. O. H.: Hong. .T. H. Tetrahedron Lett. 2002. +3. 6399. (b) Honn. T. H.: Gao. M. Y. Choi. Y.: Cheng. Y.-C.: Schinazi. R. F.: Chu. C. K. Corbohndrate Res. 2000, 328. 37; (c) Hong. J. H.: Gao, M. Y.: Chu. C. K. Tetrahedron Lett 1999, 40.231: (d) Hong. J. H.: Lee. K: Choi, Y: Chu. C. K. Tetrahedron Lett. 1988. 39, 3413.

8. For selected references for nucleosides: (a) Hong. J. H.: Shimn. M J.: Ro. B. O.: Ko. O. H. J. Org Chem. 2002. 67. 6387; (b) Ewing. D.: Glacon. V: Mackenzie. G.: Postel. D.: Len. C. Tetrohedhon Letf. 2002. 43. 3503: (c) Gurjar, M. K: Maheshwar. K. J. Ong. Chent 2001. 66,7552 ; (d) Crimmins. M. T.: King. B. W: Zuercher. W. J.: Choy. A. L. J. Ong Chem 2000, 65, 8499

9. For selected references for nucleosides: (a) Trost. B. M. Kallander. L. S. J. Org. Chem. 1999. 64. 5427: (b) Trost. B. M.: Shi. Z. J. An Chen Soc. 1996. 118. 3037: (c) Deardorft. D. R. Savin. K. A.: Justman. C. J.: Karanjawala. Z. E.: Sheppeck. II. J. E.; Hager, D. C.: Aydin. N. J. Org. Chem. 1996. 61,3616 (d) Nokami, J: Matsuura, H.: Nakasima, K: Shibata, S. Chemistry Lett 1994, 1071: (e) Evans, C. T.: Roberts, S. M. Shoberu. K. A.: Sutherland. A. G. J. Chem. Soc. Perkin Trans 1 1992.589

10. (a) Waga. T.: Ohriu. H.: Meguro. H. Nucleosides \& Nucleotides 1996. 15. 287; (b) Waga, T.: Nishizaki. T.: Miyakawa, J.: Ohriu. H.: Meguro, H. Biosci. Biotechol. Biochem. 1993, 57. 1433 .

11. Kato. K:: Suzuki. H.: Tanaka. H.: Myasakia. T.: Baba. M.: Yamaguchi. K.: Akita. H. Chem. Phom. Bull. 1999. 47. 1256.

12. (a) Haines. D. R.: Tseng. C. K. H.: Marquez. V. E. J. Med Chen. 1987. 30,943: (b) Jeong, L. S.: Lee. Y. A.: Moon. H. R.; Chun, M. W. Nucleosides \& Nucleotides 1998. 17. 1473.

13. (a) Hossain. N.: Rozenski, J:- De Clercy. E: Herdewjn. P. Tetrahedron 1996. 52. 13655: (b) Teon. G. S.: Nair. V. Tetrahedron 1996.52 .12643 .

14. (a) Gundersen. L.-L.: Bentneche. T.: Undheinn. K. Tetrahedon Letf. 1992, 33, 1085: (b) Gundersen, L.-L.: Benneche. T.: Rise. F.: Gogoll. A.: Undheim, K. Acta Chem. Scand. 1992, 46.761. 